\title{
Detection of Avian Influenza-DNA Hybridization Using Wavelength-scanning Surface Plasmon Resonance Biosensor
}

\author{
Shin Ae Kim and Sung June Kim* \\ School of Electrical Engineering and Computer Science, Seoul National University, Seoul 151-742, Korea \\ Sang Hun Lee and Tai Hyun Park \\ School of Chemical and Biological Engineering, Institute of Bioengineering, \\ Seoul National University, Seoul 151-742, Korea \\ Kyung Min Byun \\ Department of Biomedical Engineering, Kyung Hee University, Yongin 446-701, Korea \\ Sung Guk Kim \\ College of Veterinary Medicine, Cornell University, Ithaca, New York 14853, USA \\ Michael L. Shuler \\ Department of Biomedical Engineering, Cornell University, Ithaca, New York 14853, USA
}

(Received June 1, 2009 : revised July 3, 2009 : accepted August 5, 2009)

\begin{abstract}
We designed a wavelength interrogation-based surface plasmon resonance (SPR) biosensor to detect avian influenza DNA (AI-DNA). Hybridization reactions between target AI-DNA probes and capture probes immobilized on a gold surface were monitored quantitatively by measuring the resonance wavelength in the visible waveband. The experimental results were consistent with numerical calculations. Although the SPR detection technique does not require the DNA to be labeled, we also evaluated fluorescently-labeled targets to verify the hybridization behavior of the AI-DNA. Changes in resonance were found to be linearly proportional to the amount of bound analyte. A wavelength interrogation-type SPR biosensor can be used for rapid measurement and high-throughput detection of highly pathogenic AI viruses.
\end{abstract}

Keywords: SPR biosensor, Wavelength interrogation, Avian influenza virus, DNA hybridization, Label-free

OCIS codes: (240.6680) Surface plasmons; (170.0170) Medical optics and biotechnology; (310.0310) Thin films

\section{INTRODUCTION}

Avian influenza (AI) is a contagious disease that infects chickens, turkeys, and migratory birds. The sources of infection, avian influenza viruses (AIVs), are highly species-specific and rarely cross species barriers. However, when avian and human viruses contaminate an intermediate host simultaneously, AIVs produced by genetic recombination between the different strains

*Corresponding author: kimsj@snu.ac.kr may infect humans. Recently, pure AIVs with high pathogenicity, namely $\mathrm{H} 5 \mathrm{~N} 1$ and H7N7, were found to have been transmitted directly into humans [1]. Because highly pathogenic AIVs can cause fatalities in humans and lead to substantial economic losses because of infection of commercial poultry, rapid screening techniques for AIVs are in high demand [2].

Several virological methods are commonly used for virus detection; these include virus isolation, reversetranscriptase polymerase chain reaction (RT-PCR), 
and antigen-capture immunoassays [2]. Although virus isolation techniques are sensitive, they are labor-intensive and time-consuming. RT-PCR is a well-known detection approach; however, complex processes make this method inappropriate for real-time and in situ analysis. Antigen capture immunoassays, although rapid, are relatively expensive and have low sensitivity. Therefore, a simple, rapid, and sensitive detection method to overcome the limitations of conventional virological techniques is required.

Surface plasmon resonance (SPR), a surface-limited optical technique that senses refractive index changes that occur in the vicinity of a thin metal surface, has been used to measure DNA hybridization [3]. SPR biosensors have attracted significant interest because they can be used for real-time monitoring and sensitive and quantitative detection [4]. In particular, label-free measurements enable SPR technology to be used for direct detection of binding events between a target analyte and its corresponding receptor. This method is also applicable in situ because hybridization kinetics can be detected immediately on the sensor surface.

Among various SPR measurement schemes, SPR imaging systems have been explored extensively due to their potential for use in high-throughput sensing applications $[3,5,6]$. An intensity-based SPR imaging system comprising a monochromatic light source and a fixed incidence angle has a simple structure. However, this system has relatively low sensitivity and requires laborious experiments to determine the optimal incidence angle with the highest slope [7]. Thus, optical sources at near-infrared (NIR) range have been used to improve the sensitivity of SPR imaging measurements. At long wavelengths, reflectance curves sharpen significantly; a narrow SPR curve results in high sensing contrast and increased sensitivity [7]. However, the application of NIR to SPR microarray imaging is limited, because as the surface plasmon propagation length increases with wavelength, the lateral image resolution becomes significantly poorer. Moreover, accurate quantification of DNA hybridization is challenging, though SPR analysis of oligonucleotide arrays on gold surfaces can differentiate between single- and double-stranded DNA regions [3].

In this study, we employed a wavelength interrogation SPR method for quantitative detection of AI-DNA hybridization. In contrast to the angle interrogation method, wavelength scanning with no moving parts can be used to construct a compact and robust biosensor system with a microarray-based multi-channel configuration. In particular, because the plasmon waves are excited by visible wavelengths of light, the image resolution is better than that obtained using NIR wavebands. Moreover, the capture probes of thiolated oligonucleotides are bound onto a gold surface to detect AI-DNA, so that additional reactive layers and complex surface modifications are not required. Our preliminary experimental results indicate that the AIV sensor system that we designed has several advantages such as rapid measurements, high-throughput detection, and minimum reagent consumption.

\section{MATERIALS AND METHODS}

\section{Preparation of reagents and probes}

We used two different buffer solutions: immobilization buffer (1 $\left.\mathrm{M} \mathrm{KH}_{2} \mathrm{PO}_{4}\right)$ and hybridization buffer $(1 \mathrm{M} \mathrm{NaCl}, 10 \mathrm{mM}$ Tris-buffer, $\mathrm{pH}$ 7.4, and $1 \mathrm{mM}$ EDTA). Dithiothreitol (DTT), ethyl acetate, and 16-mercaptohexadecanoic acid (MCH) were purchased from Sigma-Aldrich (Saint Louis, MO, USA). The HPLC-purified capture and target oligonucleotide sequences were purchased from Genotech Inc. (Taejeon, Korea) and IDT Inc. (Coralville, IA, USA). The oligonucleotide sequences are listed below:

- Capture probe $\left(\mathbf{C}_{1}\right)$ : 35-mer oligonucleotide with a 15-mer thymine (underlined) as a spacer:

5' - Thiol - TTT TTT TTT TTT TTT ATT GGA CAC GAG ACG CAA TG - 3'

- Target probe $\left(T_{1}\right):$ 20-mer complementary oligonucleotide:

5' - CAT TGC GTC TCG TGT CCA AT - 3'

- Fluorescein-modified target probe $\left(T_{1-d y e}\right)$ :

20-mer complementary oligonucleotide with fluorescein: 5 - fluorescein - CAT TGC GTC TCG TGT CCA AT - 3'

- Mismatched target probe $\left(\mathbf{T}_{2}\right)$ : 10-base (underlined) mismatched oligonucleotide: 5' - CAT TGC GTC TGC ACA GGT TA - 3'

- Fluorescein-modified mismatched target probe $\left(\mathbf{T}_{2-\text {-dye }}\right)$ : 10-base (underlined) mismatched oligonucleotide labeled with fluorescein:

5 - fluorescein - CAT TGC GTC TGC ACA GGT TA - 3'

The thiol-labeled capture probe $\left(\mathrm{C}_{1}\right)$ was immobilized and a gold-S (from thiol) covalent linkage was formed on a gold surface $[5,6,8,9]$. Target probes $\left(\mathrm{T}_{1}\right.$ and $\left.\mathrm{T}_{1 \text {-dye }}\right)$ were designed with a sectional sequence of avian influenza A virus (A/avian/NY/73-63-6/00(H7N2)) hemagglutinin. All stock oligonucleotide solutions were made with deionized distilled water (DDW) and stored at $-20^{\circ} \mathrm{C}$.

\section{Preparation of SPR sensor chips}

A gold film with a thickness of $45 \mathrm{~nm}$ was sputtered onto a $20 \times 20 \times 1 \mathrm{~mm}$ thick SF10 slide glass substrate after evaporation of a 5-nm thick chromium adhesion layer. The gold surface was cleaned sequentially in acetone and $70 \%$ ethanol solution for 10 minutes in a sonication bath. The chip was then rinsed with DDW 
and dried by blowing nitrogen. Finally, the sensor chip was irradiated by UV-light to remove organic residues.

\section{SPR experiments}

We used a custom-made Kretschmann-configuration optical setup to detect SPR signals. As shown in Fig. $1(\mathrm{a})$, the incidence angle was fixed at $6^{\circ}$ for wavelength scanning measurements. The SPR chip was index-matched to a SF10 prism. Two PDMS microfluidic channels (working and reference channels) were placed on top of the chip and screw-clamped to seal the channels. TM-polarized light with a bandwidth of $0.1 \mathrm{~nm}$ was incident when a white light from Xe-lamp (66901, Oriel) passed through a monochromator (7400, Oriel) and an optical polarizer. The reflected light intensity was measured with a CCD camera (XCD-X710CR, Sony) in the wavelength range from 550 to $650 \mathrm{~nm}$. The resonance wavelength was determined by fitting the reflectance curve to a second-order polynomial equation. Before SPR measurement, the reflectance characteristics of TE-polarized light were recorded to eliminate common-mode noise components arising from the wavelength of light source. We obtained SPR curves by calculating the difference in reflectance

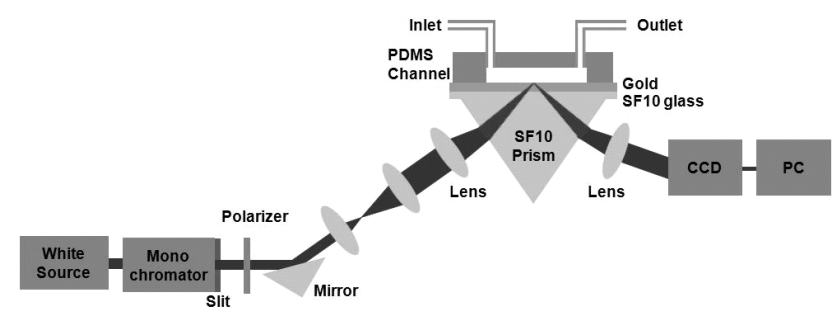

(a)

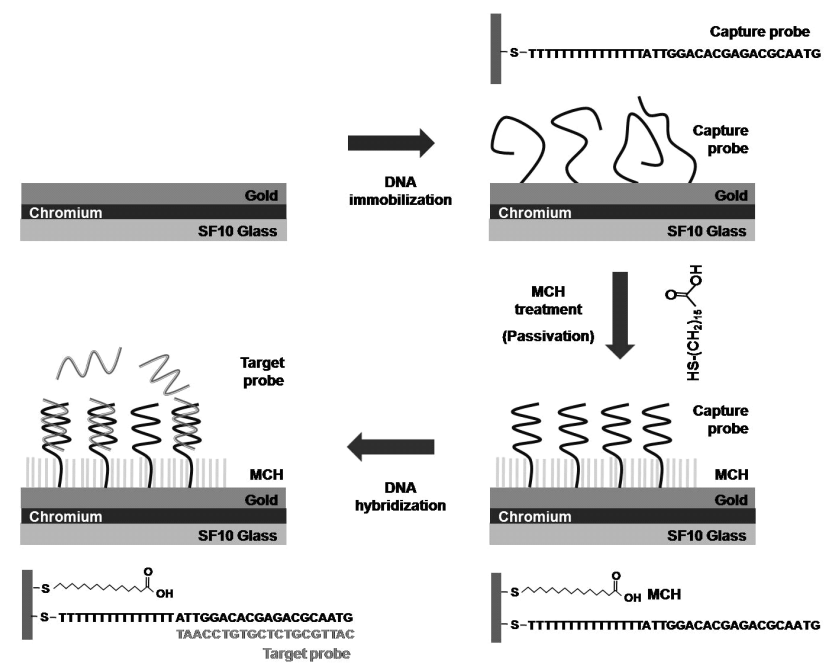

(b)

FIG. 1. (a) Schematic diagram of the wavelength interrogation-based SPR measurement system. (b) Illustration of capture probe immobilization, $\mathrm{MCH}$ treatment, and target AI-DNA hybridization on a SPR sensor chip. between TM- and TE- polarized incidences. While the results of ethanol experiments [10] are not shown here, the minimum value of measurable refractive index change was determined to be $1.6 \times 10^{-4}$ in refractive index units. All SPR experiments were carried out at room temperature with a flow rate of $2 \mu \mathrm{l} / \mathrm{min}$ through a syringe pump (SP230IW, WPI).

\section{Numerical calculation}

Rigorous coupled-wave analysis (RCWA) has been employed in numerical simulations to calculate the optical responses of DNA hybridization [11]. RCWA has also been successfully used to describe SPR experiments $[12,13]$. Convergence in computation was accomplished by including a sufficient number of spatial harmonics orders (more than 30) at the expense of prolonged computation time. In the numerical model, a TM-polarized plane wave with a fixed incidence angle of $60^{\circ}$ illuminated a SPR sensor chip. Binding analytes that cover a gold surface were modeled as a 3 -nm thick homogeneous dielectric layer. As shown in Fig. 1(b), because target DNAs coupled with capture probes on a sensor surface do not have a significant effect on the thickness of the binding layer, the shift in the resonance wavelength was assumed to be created by changes in the refractive index at the sensor surface. Optical constants of gold, chromium, and SF10 glass were taken from Ref. 14.

\section{EXPERIMENTS AND DISCUSSION}

The construction of a capture DNA-MCH layer and subsequent hybridization reactions are illustrated in Fig. 1(b). To immobilize thiol-modified oligonucleotides on the gold surface, thiol functional groups were treated with a $1 \mathrm{M}$ DTT-containing reaction buffer and any remaining DTT was removed by extraction with $100 \mu \mathrm{l}$ of ethyl acetate and vacuum evaporation. The gold sensor chip was then immediately incubated in 2 $\mu \mathrm{M}$ capture probe $\left(\mathrm{C}_{1}\right)$ in an immobilization solution for 12 hours. After incubation, the substrate was washed with DDW. Because thiolated capture probes alone are not able to form a surface with uniform coverage due to non-specific interactions between DNA and the gold surface, $1 \mathrm{mM} \mathrm{MCH}$ was used to improve immobilization efficiency and displace non-specific interactions [5,9]. Use of $\mathrm{MCH}$ resulted in better orientation of the capture probe layer and a notable increase in hybridization efficiency. The sensor chip was then soaked in DDW and applied to an SPR measurement system to monitor the hybridization reaction in real-time.

Before the DNA hybridization experiment, the concentrations of capture probe on the gold film and sodium chloride in the hybridization buffer were optimized. If 
the capture probes pack too closely, hybridization is inefficient due to steric hindrance and/or electrostatic interactions [8]. Moreover, an increase in cations in hybridization environments may stabilize hybridization reactions because capture and target probes that are negatively charged may repulse one another [8]. Two $\mu \mathrm{M}$ of capture probe and $1 \mathrm{M}$ of $\mathrm{NaCl}$ in the hybridization solution were determined to be optimal.

To detect AI-DNA hybridization using SPR detection, the SPR characteristics of the hybridization interactions were first measured. $\mathrm{T}_{1}$ AI target probes $(3 \mu \mathrm{M})$ were injected through micro-fluidic channels in which thiololigonucleotide $\mathrm{C}_{1}$ probes had been immobilized on a gold surface. The resonance wavelength was $635.0 \mathrm{~nm}$ before hybridization and $637.4 \mathrm{~nm}$ after hybridization; thus, the resonance shift was $2.4 \mathrm{~nm}$ (Fig. 2). From RCWA calculations, this resonance shift corresponds to a change in the refractive index of dielectric layers formed at the sensor surface of between 1.4021 to 1.4347, assuming that the thickness is fixed at $3 \mathrm{~nm}$. The measured SPR curves matched the numerical results well (Fig. 2).

Next, to explore specific binding reactions, hybridization was repeatedly performed by injecting a total volume of $500 \mu \mathrm{l}$ of hybridization solution containing hybridization buffer and $1 \mu \mathrm{M}$ target probes of $\mathrm{T}_{1}$ and $\mathrm{T}_{2}$. The microfluidic channels were washed with hybridization buffer with no probe to remove unoccupied $\mathrm{C}_{1}$, and MCH to stabilize the baseline before the hybridization reaction. It is clear from Fig. 3(a) that a reaction occurred in the working channel into which the solution with target $T_{1}$ was injected, while no significant changes were observed when the mismatched target $T_{2}$ was injected. This indicates that $\mathrm{T}_{1}$ probes hybridized to immobilized $\mathrm{C}_{1}$ capture probes and were specifically adsorbed on the sensor surface.

Fluorescent microscopic images of DNA hybridi-

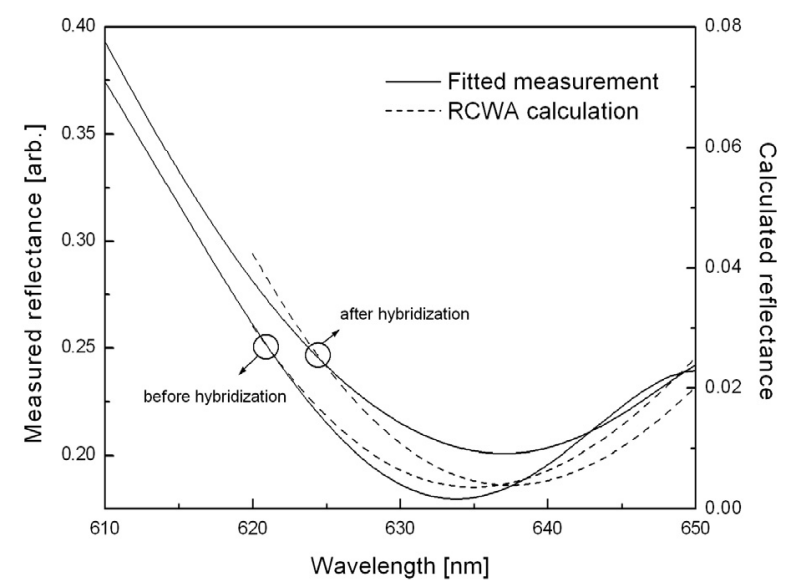

FIG. 2. SPR reflectance curves from numerical calculations (dotted lines) and experimental results (solid lines) before and after AI-DNA hybridization. zation were monitored using two fluorescein-modified target probes $\left(\mathrm{T}_{1 \text {-dye }}\right.$ and $\left.\mathrm{T}_{2 \text {-dye }}\right)$ using blue light for fluorescence excitation. After the identical binding procedures described above, an apparent difference in fluorescence was observed between the two target probes (see Fig. 3(b)). A green fluorescent signal was obtained when $\mathrm{T}_{1 \text {-dye }}$ probes were hybridized to the $\mathrm{C}_{1}$ capture probes, providing further evidence that the binding of $T_{1}$ to the capture probes is specific.

Subsequently, $\mathrm{T}_{1}$ target probes at various concentrations were hybridized with immobilized $\mathrm{C}_{1}$ probes to evaluate the linear sensing performance of the SPR system. For wavelength interrogation-based SPR detection to be useful quantitatively, it is important that the change in resonance wavelength be linearly proportional to the concentration of adsorbed double-strand AI-DNA on the sensor surface, and thus the amount of bound analyte. Figure 4(a) shows the experimental change in SPR wavelength when $T_{1}$ was diluted in the hybridization buffer to a concentration of 1 to $5 \mu \mathrm{M}$. Mean values with error bars ffi standard error (standard error=S.D. $/(\# \text { of samples })^{1 / 2}$ ) were calculated from three individual hybridization experiments, and the linear fit to the experimental data is indicated by the

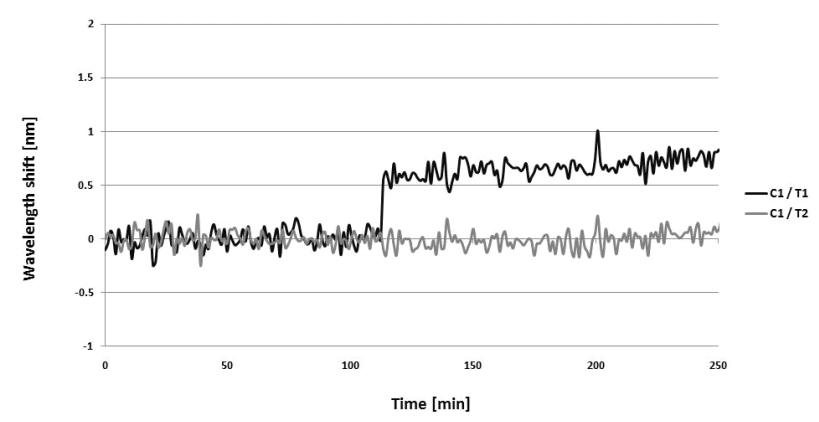

(a)
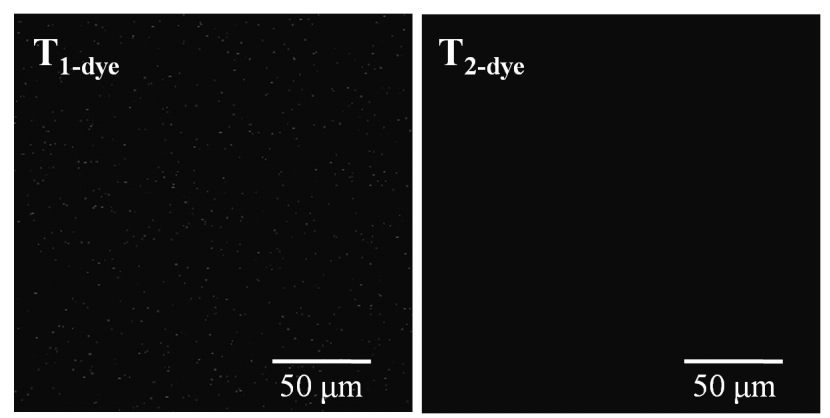

(b)

FIG. 3. (a) Specific response to matched and mismatched target probes when immobilized capture probes on a sensor surface are exposed to complementary $\left(\mathrm{T}_{1}\right.$, black line) or non-complementary $\left(\mathrm{T}_{2}\right.$, gray line) target probes. (b) Fluorescent microscopic images after AI-DNA hybridization between capture $\left(\mathrm{C}_{1}\right)$ and target ( $\mathrm{T}_{1 \text {-dye }}$ and $\left.\mathrm{T}_{2 \text {-dye }}\right)$ probes. 


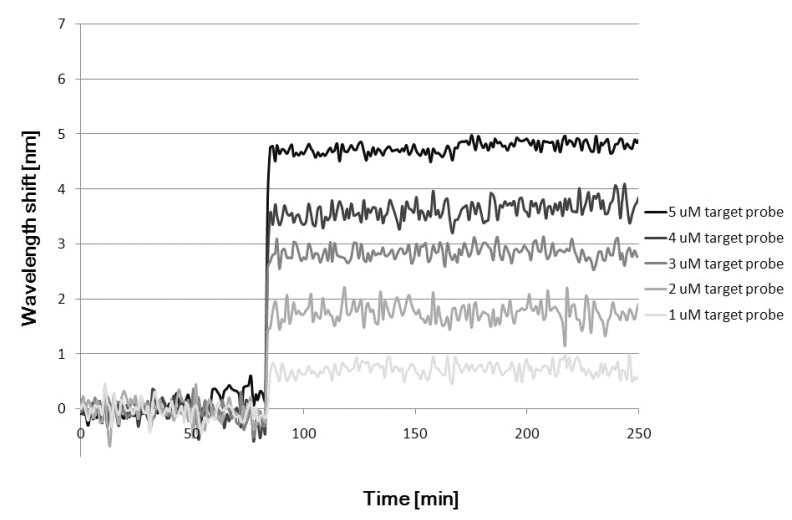

(a)

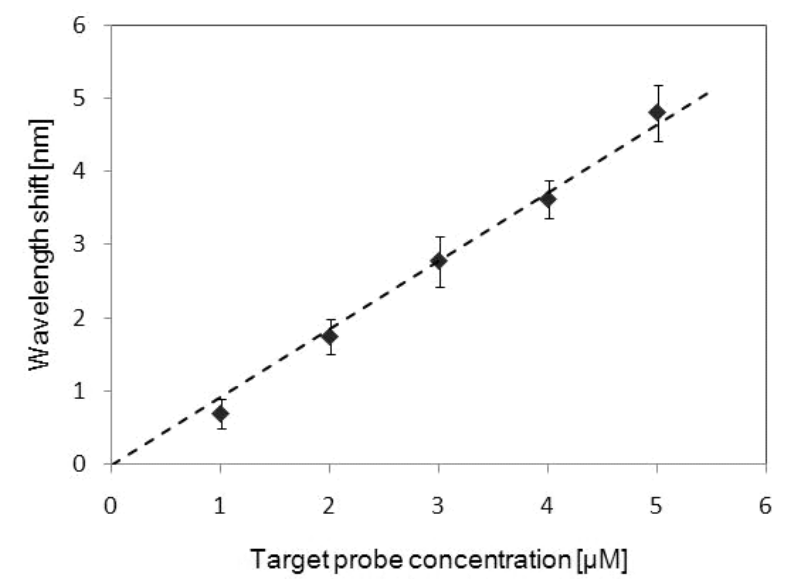

(b)

FIG. 4 (a) SPR responses of AI-DNA hybridization when the concentration of target probe was varied from 1 to $5 \mu \mathrm{M}$. (b) Shift in resonance wavelength after AI-DNA hybridization experiments and linear regression analysis of this shift (dashed line).

dashed line in Fig. 4(b). The resonance wavelengths for 1 to $5 \mu \mathrm{M}$ probe were $0.69 \pm 0.31 \mathrm{~nm}, 1.74 \pm 0.37 \mathrm{~nm}$, $2.77 \pm 0.68 \mathrm{~nm}, 3.62 \pm 0.39 \mathrm{~nm}$ and $4.80 \pm 0.57 \mathrm{~nm}$, respectively. Using the linear function $y=a x$, the proportional constant $a$ was calculated as 0.93. Linear regression analyses showed that the change in resonance with an increasing concentration of target probe was strongly linear with $R^{2}=0.99$, where $R$ is the correlation coefficient that denotes the linearity obtainable in sensor performance. AI-DNA hybridization showed a linear relationship over a broad range of resonance wavelengths. As a nominal resolution of monochromator with the light source is $0.50 \mathrm{~nm}$, minimum detectable change of target probe concentration is estimated to be in the range of $0.46 \mu \mathrm{M}$ for our SPR setup. The detection limit of the system can be easily improved by employing a light source and monochromator with enhanced wavelength resolution or using a more sensitive detector.

Our experiments indicate that the wavelength interrogation-based SPR system can be used to quantitatively measure a large range of AI-DNA hybridization reactions. In future work, we plan to examine multi-channel detection of AI-DNA interactions using an SPR sensor chip that incorporates microarray structures. Moreover, metallic nanostructure-mediated SPR biosensors will be explored for highly sensitive detection of DNA hybridization.

\section{CONCLUSIONS}

We confirmed that a wavelength-scanning type SPR biosensor can be used for simple, label-free, and quantitative detection of AI-DNA hybridization. DNA hybridization experiments with no complex surface modification are particularly advantageous for rapid screening of AI-DNA. Concentrations of target and capture probes and reagents were optimized for efficient analysis of oligonucleotide DNA sequences obtained from a highly pathogenic influenza subtype. Our AI-DNA hybridization reactions, performed using visible wavelengths of light, were highly specific and linear over a broad range of binding events. Furthermore, our experimental results were consistent with numerical calculations based on RCWA methods. The combination of a wavelength interrogation-based SPR system and microarrays on a sensor surface will allow the realization of high-throughput DNA hybridization biosensors.

\section{ACKNOWLEDGMENT}

This work was supported by the international collaboration program NBS-ERC of MEST/KOSEF (R11-2000075-00002-0). K. M. Byun would like to thank the Korea Research Foundation Grant of MOEHRD (Basic Research Promotion Fund, KRF-2008-331-D00229) for funding support.

\section{REFERENCES}

1. K. Hidari, S. Shimada, Y. Suzuki, and T. Suzuki, "Binding kinetics of influenza viruses to sialic acid-containing carbohydrates," Glycoconj. J. 24, 583-590 (2007).

2. J. Xu, D. Suarez, and D. S. Gottfried, "Detection of avian influenza virus using an interferometric biosensor," Anal. Bioanal. Chem. 389, 1193-1199 (2007).

3. A. J. Thiel, A. G. Frutos, C. E. Jordan, R. M. Corn, and L. M. Smith, "In situ surface plasmon resonance imaging detection of DNA hybridization to oligonucleotide arrays on gold surfaces," Anal. Chem. 69, 4948-4956 (1997).

4. J. Homola, S. S. Yee, and G. Gauglitz, "Surface plasmon resonance sensors: review, Sens. Actuators B 54, 3-15 (1999).

5. M. G. Manera, J. Spadavecchia, A. Leone, F. Quaranta, R. Rella, D. Dell'arri, M. Minunni, M. Mascini, and P. 
Siciliano, "Surface plasmon resonance imaging technique for nucleic acid detection," Sens. Actuators B 130, 82-87 (2008).

6. W. Y. Chen, W. P. Hu, Y. D. Su, A. Taylor, S. Jiang, and G. L. Chang, "A multisport DNA chip fabricated with mixed ssDNA/oligo(ethylene glycol) self-assembled monolayers for detecting the effect of secondary structures on hybridization by SPR imaging," Sens. Actuators B 125, 607-614 (2007)

7. K. M. Byun, M. L. Shuler, S. J. Kim, S. J. Yoon, and D. Kim, "Sensitivity enhancement of surface plasmon resonance imaging using periodic metallic nanowires," J. Lightwave Technol. 26, 1472-1478 (2008).

8. M. Mir and I. Katakis, "Target label-free, reagentless electrochemical DNA biosensor based on sub-optimum displacement," Talanta 75, 432-441 (2008).

9. A. B. Steel, T. M. Herne, and M. J. Tarlov, "Electrochemical quantitation of DNA immobilized on gold,"
Anal. Chem. 70, 4670-4677 (1998).

10. K. M. Byun, S. J. Yoon, D. Kim, and S. J. Kim, "Experimental study of sensitivity enhancement in surface plasmon resonance biosensors by use of periodic metallic nanowires," Opt. Lett. 32, 1902-1904 (2007).

11. M. G. Moharam and T. K. Gaylord, "Diffraction analysis dielectric surface-relief gratings,” J. Opt. Soc. Am. 72, 1385-1392 (1982).

12. J. Cesario, R. Quidant, G. Badenes, and S. Enoch, "Electromagnetic coupling between a metal nanoparticle grating and a metallic surface," Opt. Lett. 30, 3404-3406 (2005).

13. Y. Kanamori, K. Hane, H. Sai, and H. Yugami, "100 $\mathrm{nm}$ period silicon antireflection structures fabricated using a porous alumina membrane mask," Appl. Phys. Lett. 78, 142-143 (2001).

14. E. D. Palik, Handbook of Optical Constants of Solids (Academic Press, Orlando, FL, USA, 1985). 